\title{
Die Energiewende: eine Herausforderung für die Chemie
}

\author{
Leitartikel \\ Robert Schlögl \\ Department of Inorganic Chemistry, Fritz Haber Institute of the Max Planck Society, \\ Faradayweg 4-6, 14195 Berlin, Germany
}

Die Energiewende hat in Deutschland einen Impuls für den nachhaltigen Umbau des Energiesystems gesetzt. Als Ersatz für den kurzfristigen Ausfall der Kernspaltungsenergie werden die verstärkte Nutzung regenerativer Stomerzeugung und Einsparung an Primärenergietragern empfohlen.

Fossile Energieträger sind stofflicher Natur und lassen sich daher speichern. Sie stamen aus der Photosynthese, die Kohlendioxid und Wasser zu Zucker und in Nachfolgeprozessen zu Polymeren mit erheblichen Sauerstoffanteilen umsetzt. Dieser Gehalt begründet die vergleichsweise geringe Energiedichte nachwachsender Energieträger, die nicht mit der Nahrungsmittelproduktion konkurrieren. Biomaterialien auch der dritten Generation werden kaum mehr als zehn Prozent des Primärenergiebedarfes abdecken. So sind wir zwingend auf regenerative Energie als primäre Elektrizität angewiesen.

Das heute notwendige Vorhalten entsprechender fossiler Schattenkapazitäten ist keine Lösung, wenn wir die formulierten Nachhaltigkeitsziele erreichen wollen.

Dabei ist es sehr sinnvoll, Primärelektrizität aus regenerativen Quellen zu gewinnen, da so ein Energiesystem den maximalen Wirkungsgrad erzielt. Auch eine noch so ausgeklügelte Bedarfssteuerung kann allerdings dunkle Flauten nicht überbrücken.

Dieses Problem kann die Speicherung solarer Energie in chemischen Bindungen sehr überzeugend lösen. Der primär gebildelte Energieträger Wasserstoff lässt sich in weitere solare Brennstoffe umwandeln. Sie können grundsätzlich alle Anforderungen einer modernen Energieinfrastruktur erfüllen. Wegen des Wirkungsgrads sollte die Zahl der chemischen Umwandlungsschritte so klein wie möglich bleiben. Wasserstoff kommt in Frage, während Träger wie Methan, Methanol, Ammoniak oder Designerkraftstoffe dezentrale Anwendungen bedienen.

Damit reduziert sich das Problem auf die effiziente Umsetzung von Wasser, Wasserstoff, Kohlendioxid und
Stickstoff zu Energieträgern. Diese im Labormaßstab sehr gut lösbaren Aufgaben entwickeln sich zu enormen Herausforderungen, man denke nur an die entsprechenden Dimensionen von Anlagen und benötigten Materialien sowie an die unbedingt zu minimierende Zahl von Hilfsschritten in den Prozessen für die Reinigung von Edukten und Produkten.

Leider ist unser Wissen um die Optimierung der nötigen katalytischen Schritte weder in den Grundlagen noch in der Prozessrealisation so weit gereift, dass sich eine Aufgabe dieser Größenordnung durch das Aufskalieren heute verfügbarer Demonstratortechnik lösen lässt.

Die Energiewende zwingt uns dazu, die Katalysewissenschaft soweit voranzutreiben, dass wir kleine Moleküle hocheffizient umwandeln können. Die Chemie, die sich gerne als von der Energieversorgung abhängig darstellt, wird damit zur antreibenden Wissenschaft und Technik. Wir müssen nicht nur in akademischen Übungen nach neuen Ansätzen suchen, sondern auch die Anstrengungen verstärken, um die Grundlagen der Katalyse $\mathrm{zu}$ beherrschen. Dann sind wir in der Lage, die Reaktionen kleiner Moleküle so zu steuern, dass wir nachhaltig mit den benötigten Stoffmengen sicher und wirtschaftlich umgehen können.

Wir verfügen grundsätzlich über die nötigen wissenschaftlichen Mittel für diese große Aufgabe, sollten sie aber durch Arbeitsteilung und Vernetzung wirksamer einsetzen. Die Herausforderung der Energiewende ist ähnlich der Mondlandung und sollte mit ebenso großen Engagement vorangetrieben werden. Dabei erhält die Chemie eine einmalige Chance, sich für jeden erkennbar als fundamental benötigte Wissenschaft jenseits des Images einer Hilfswissenschaft zu positionieren. Wir sollten diese Chance beherzt nutzen. 